\title{
Vitamin D deficiency as adverse drug reaction? A cross-sectional study in Dutch geriatric outpatients
}

\author{
A. C. B. van Orten-Luiten ${ }^{1,2}$ (D) - A. Janse ${ }^{2}$ - R. A. M. Dhonukshe-Rutten ${ }^{3}$. \\ R. F. Witkamp ${ }^{1}$
}

Received: 19 November 2015 / Accepted: 19 January 2016 / Published online: 12 February 2016

(C) The Author(s) 2016. This article is published with open access at Springerlink.com

\begin{abstract}
Purpose Adverse drug reactions as well as vitamin D deficiency are issues of public health concern in older people. However, relatively little is known about the impact of drug use on vitamin D status. Our primary aim is to explore associations between drug use and vitamin D status in older people. Furthermore, prevalences of drug use and vitamin D deficiency are estimated.

Methods In a population of 873 community-dwelling Dutch geriatric outpatients, we explored the cross-sectional relationships of polypharmacy ( $\geq 5$ medications concomitantly used), severe polypharmacy ( $\geq 10$ medications), and use of twentyone specific drug groups, with serum 25 -hydroxyvitamin D $(25(\mathrm{OH}) \mathrm{D})$ by analysis of covariance.
\end{abstract}

Electronic supplementary material The online version of this article (doi:10.1007/s00228-016-2016-2) contains supplementary material, which is available to authorized users.

\section{A. C. B. van Orten-Luiten \\ wout.vanorten-luiten@wur.nl \\ R. F. Witkamp \\ renger.witkamp@wur.nl \\ A. Janse \\ jansea@zgv.nl \\ R. A. M. Dhonukshe-Rutten rosalie.dhonukshe-rutten@wur.nl}

1 Division of Human Nutrition, Pharmacology and Nutrition, Wageningen University, P.O. Box 8129, 6700 EV

Wageningen, The Netherlands

2 Department of Geriatric Medicine, Gelderse Vallei Hospital, Willy Brandtlaan 10, 6716 RP Ede, The Netherlands

3 Division of Human Nutrition, Wageningen University, P.O. Box 8129, 6700 EV Wageningen, The Netherlands
Results Overall prevalence of polypharmacy was $65 \%$, of severe polypharmacy $22 \%$. Depending on the cut-off value, prevalence of vitamin D deficiency was $49 \%(<50 \mathrm{nmol} / \mathrm{l})$ or $77 \%(<75 \mathrm{nmol} / \mathrm{l})$. Of the patients using a vitamin D supplement, $17 \%(<50 \mathrm{nmol} / \mathrm{l})$ or $49 \%(<75 \mathrm{nmol} / \mathrm{l})$ were still deficient. In non-users of supplemental vitamin $\mathrm{D}$, after adjustment for age and gender, negative associations were found for severe polypharmacy, metformin, sulphonamides and urea derivatives (SUDs), vitamin $\mathrm{K}$ antagonists, cardiac glycosides, loop diuretics, potassium-sparing diuretics, ACE inhibitors, and serotonin reuptake inhibitors; for non-selective monoamine reuptake inhibitors (NSMRIs) the association was positive. The most extreme impacts of drug use on adjusted mean 25(OH)D were $-19 \mathrm{nmol} / \mathrm{l}$ for SUDs and $+18 \mathrm{nmol} / \mathrm{l}$ for NSMRIs.

Conclusion Drug use should be considered a risk factor for vitamin D deficiency amongst geriatric outpatients.

Keywords Adverse drug reaction · Drug-food interaction . Vitamin D · Polypharmacy · Elderly

\section{Introduction}

Adverse drug reactions (ADRs) substantially contribute to global disease burden. Median prevalence of ADR-related hospital admissions in European countries is estimated $3.5 \%$, prevalence of ADRs amongst inpatients $10.1 \%$ [1]. These figures increase significantly with increase in age [2].

The high prevalence of vitamin D deficiency in older people is also an issue of global public health concern [3]. Apart from a negative impact on calcium metabolism and bone health, vitamin $\mathrm{D}$ deficiency might also lead to non-skeletal diseases and an increased inflammatory status: [4, 5]. An important determinant of 
deficiency of this micronutrient is a reduced synthesis of precursors in the skin by increase in age, less exposure to sunlight, or pigmented skin. Furthermore, insufficient dietary intake, impaired absorption, liver or kidney dysfunction, obesity, and inflammation may be of influence [6-10]. However, about the role of medication little is known. Observational and experimental research on this subject is scarce [11]. Also during drug development and in post-marketing studies, drug effects on nutritional status are not specifically addressed [12]. This is remarkable, as drug use as well as an impaired nutritional status are related to frailty [13-15] and predict disability, hospitalisations, and death [16].

Challenged by the lack of knowledge of this type of adverse drug reaction, we formulated the following study objectives: to investigate cross-sectional relationships of polypharmacy, number of drugs used, and use of 21 individual drug groups with serum 25hydroxyvitamin D $(25(\mathrm{OH}) \mathrm{D})$; secondary aims were to determine prevalences of drug use and vitamin $\mathrm{D}$ deficiency. For statistical analyses we used data from a population of community-dwelling geriatric outpatients of a Dutch, non-academic, regional hospital.

\section{Methods}

\section{Study population}

Our original study population consisted of 892 community-living outpatients of the department of Geriatric Medicine of Gelderse Vallei Hospital (Ede, The Netherlands) at their first visit of the outpatient clinic, from August 2011 until January 2013. Indications for consultation were cognitive problems (60\%), falls (14\%) or other (24\%). Patient data were registered in the electronic patient file system Norma EPD. After exclusion of subjects either younger than 55 years or not having $25(\mathrm{OH}) \mathrm{D}$ measured, data of 783 patients were left for initial analysis. In a subgroup of 631 patients not using a vitamin D supplement, associations between drug use and serum vitamin $\mathrm{D}$ were explored in a simple regression model. Because of missing data, another 29 subjects were omitted in the complex model $(N=602)$. According to the Dutch Medical Research Involving Human Subjects Act (WMO) no ethical approval was needed, since this study involved retrospective analysis of anonymised patient data only.

\section{Data}

History, physical examination and other clinical assessments of the geriatric patients were conducted by the medical staff, assisted by trained nurses. Non-fasting blood samples were collected at the Gelderse Vallei Hospital on the day of first visit. Assays were conducted by the hospital laboratory once a week. Vitamin D status was measured using the 25-OH-Vitamin D3/D2 Reagent Kit for HPLC analysis according to the manufacturer's instructions (Chromsystems Instruments \& Chemicals, Gräfelfing, Germany). For more details on $25(\mathrm{OH}) \mathrm{D}$ assessment we refer to the electronic supplementary material. Standardised anthropometric measurements were conducted by trained nurses. Weight was determined to the nearest $0.1 \mathrm{~kg}$ through a digital floor scale (Seca 770), height to the nearest $0.1 \mathrm{~cm}$ using a telescopic height rod (Seca 220). If patients had difficulties with standing, only weight was measured using a chair scale (Seca D94-09-033). Assessment of drug use was based on both prescribed and over-the-counter drugs the patients were asked to bring to the clinic, on (hetero)anamnesis, and on medication lists from pharmacies, if available. Non-compliance was structurally asked for. The Mini Nutritional Assessment (MNA) was performed in patients aged 65 and older. Collected patient data were registered in the hospital electronic patient file system Norma/NeoZis according to hospital standards.

Raw patient data were extracted from the patient files and further processed. The day of blood collection was recorded into the variable season: winter (October-March) or summer (April-September). Education attainment was classified as either primary school ( $\leq 6$ years) or post-primary education and higher ( $>6$ years), smoking as never, ever, and current. Categorisation of alcohol use was based on the Alcohol Consumption Index according to Garretsen: not/light, moderate, excessive/very excessive [17]. Medication and supplements were coded by the Anatomic Therapeutical Classification (ATC) index. Polypharmacy was defined as the concomitant use of five or more different ATC-coded substances; severe polypharmacy for ten or more. Medications of interest were drug groups at ATC level 4 used by minimally $10 \%$ of the patients: proton pump inhibitors (PPIs), osmotically active laxatives, biguanides, sulfonamides and urea derivatives (SUDs), vitamin K antagonists, platelet aggregation inhibitors, thiazide diuretics, loop diuretics, selective beta-blocking agents, dihydropyridines, angiotensin-converting enzyme (ACE) inhibitors, angiotensin-2 antagonists, statins, anilides, non-selective monoamine reuptake inhibitors (NSMRIs), and selective serotonin reuptake inhibitors (SSRIs). For a more complete comparison with the results of a similar cross-sectional study, also cardiac glycosides, benzodiazepines, oral antidiabetics, potassium-sparing diuretics, and antidepressants were investigated [18]. After being processed, data were ready to be analysed. 


\section{Statistical analysis}

Statistical analyses were conducted with IBM SPSS statistics Version 20. Because of non-normal distributions, numerical data were reported as medians with interquartile range (IQR); categorical data as prevalences. Medians were compared by the nonparametric median test, differences between prevalences by chi-squared tests. Analysis of covariance (ANCOVA) was applied to investigate the associations between the dependent variable $25(\mathrm{OH}) \mathrm{D}$, and the independent variables 'number of ATC-coded substances', polypharmacy, severe polypharmacy, and twenty-one individual drug groups in subjects not taking a specific vitamin D supplement. Use of medication was compared to non-use.

Two regression models were constructed for each drug group in non-users of supplemental vitamin D. Firstly, a simple regression model with age and gender as confounders, and secondly, a complex model that also adjusts for BMI, MMSE score, and use of multivitamins. Pragmatically, one complex model was created for all drug groups by using 'number of ATC-coded substances used' as independent variable. Based on literature, the following variables were considered as extra, potential confounders: BMI, season, plasma creatinine, plasma albumin, MMSE score, education, smoking, use of alcohol, use of a vitamin D supplement, and use of a multivitamin supplement $[18,19]$. Variables were included in the model if they changed the unstandardised regression coefficient $\geq 10 \%$ after being added (linear regression, stepwise method).

To meet the assumption of normal distribution of the dependent variable and its residuals, square-root transformation of 25(OH)D was applied. Normality was checked through histograms, normal Q-Q plots, and detrended Q$\mathrm{Q}$ plots. Since associations between medication and square-root transformed $25(\mathrm{OH}) \mathrm{D}$ values and differences in means of such a variable are difficult to interpret, the adjusted means of transformed 25(OH)D were squared (back transformation) to calculate the easy to interpret mean untransformed 25(OH)D levels of users and nonusers of a medication. The difference in adjusted means $25(\mathrm{OH}) \mathrm{D}$ between users and non-users of a specific drug group is equivalent to the regression coefficient beta $(\beta)$. This is important to realise as a regression coefficient, difference, or confidence interval of a square-root transformed variable cannot be squared as back transformation [20]. The assumption homogeneity of variance was tested with the Levene's test. In case of significance, we double-checked this assumption with the variance ratio test, as in large groups small differences in variance may still result in a significant Levene's test because of increased power of this test. Effect modification by the individual covariates was tested by assessment of statistical significance of the interaction terms medication* covariate for each of the drug groups of interest. In case of significant interaction ( $p$ value $<0.1)$, analyses were stratified. Statistical tests were two-tailed and, apart from the testing of interaction, a $p$ value $<0.05$ was the criterion for statistical significance.

\section{Results}

\section{Characteristics}

In Table 1 the characteristics of 783 geriatric outpatients are presented. Median number of medications used was 6 [IQR 3-9], prevalence of polypharmacy $65 \%$, of severe polypharmacy $22 \%$. Depending on the cut-off value used, prevalence of vitamin $\mathrm{D}$ deficiency was $49 \%(25(\mathrm{OH}) \mathrm{D}<50 \mathrm{nmol} / \mathrm{l})$ or $77 \%(<75 \mathrm{nmol} / \mathrm{l})$. Of the 152 patients using a vitamin $\mathrm{D}$ supplement, a considerable number were still deficient: $17 \%$ at $<50 \mathrm{nmol} / 1$ and $49 \%$ at $<75 \mathrm{nmol} / 1$, respectively and of the 631 non-users, 57 and $83 \%$, respectively. In the severe polypharmacy subgroup of these non-users, prevalence of deficiency was 73 and $88 \%$, respectively. Compared to the supplement users, the non-users were younger ( $\geq 80$ years: $44 \%$ versus $59 \%, p<0.01$ ), had a lower risk of malnutrition (MNA screening score 12-14: $62 \%$ versus $45 \%, p<0.01)$, and used fewer medications (median [IQR]: 5 [3-8] versus 9 [6-12], $p<0.01$ ).

\section{Associations between drug use and vitamin D level}

Table 2 shows the associations between drug use and serum 25(OH)D adjusted for age and gender, in patients not using a vitamin D supplement $(n=631)$. Associations are expressed as $V$-beta $_{\text {medication }}(\beta)$. For the complete results of the further adjustments in the complex model we refer to Table 3 in the electronic supplementary material of the online version of this article.

The majority of statistical significant associations were inverse associations. In the simple model, adjusting for age and gender, we found negative associations for the number of medications used, polypharmacy, severe polypharmacy, use of oral antidiabetics, metformin, SUDs, vitamin $\mathrm{K}$ antagonists, cardiac glycosides, loop diuretics in subjects age $\geq 80$ years, potassium-sparing diuretics, ACE inhibitors, and the antidepressive SSRIs. By contrast, the association with the antidepressive NSMRIs was positive in subjects $<80$ years. The most extreme differences between adjusted mean $25(\mathrm{OH}) \mathrm{D}$ level of users and non-users of a medication were $-14.5 \mathrm{nmol} / 1$ for SUDs and $+17.5 \mathrm{nmol} / 1$ for NSMRIs. 
Table 1 Characteristics of a population of 783 Dutch geriatric outpatients, aged 55 years and older

\begin{tabular}{|c|c|c|c|c|c|}
\hline Characteristic & Category & $\begin{array}{l}\text { Percentage or } \\
\text { median [IQR] }\end{array}$ & Characteristic & Category & $\begin{array}{l}\text { Percentage or } \\
\text { median [IQR] }\end{array}$ \\
\hline \multirow[t]{2}{*}{ Gender } & male & 40.1 & MMSE (score) & & 24 [19-27] \\
\hline & female & 59.9 & & $0-18$ & 22.1 \\
\hline \multirow[t]{2}{*}{ Age (year) } & & $79[73-84]$ & & $19-24$ & 31.4 \\
\hline & $\geq 80$ & 46.9 & & $25-30$ & 46.5 \\
\hline \multirow[t]{4}{*}{ BMI (kg/m2) } & & $26.5[23.6-29.7]$ & Smoking status & never & 64.4 \\
\hline & $<21.0$ & 9.4 & & former & 25.0 \\
\hline & $21.0-26.9$ & 43.8 & & current & 10.6 \\
\hline & $\geq 27.0$ & 46.8 & Serum 25(OH)D (nmol/l) & & $50[33-72]$ \\
\hline \multirow[t]{4}{*}{ MNA screening ${ }^{\mathrm{a}}$ (score) } & & $12[10-13]$ & & $<50$ & 49.4 \\
\hline & $0-7$ & 7.9 & & $50-74$ & 27.1 \\
\hline & $8-11$ & 33.4 & & $\geq 75$ & 23.5 \\
\hline & $12-14$ & 58.7 & Medications ${ }^{\mathrm{d}}$ (number) & & $6[3-9]$ \\
\hline \multirow[t]{3}{*}{ Alcohol use ${ }^{c}$} & not/light & 85.3 & & use $(\geq 1)$ & 95.1 \\
\hline & moderate & 12.0 & & polypharmacy $(\geq 5)$ & 64.9 \\
\hline & excessive & 2.7 & & severe polypharmacy $(\geq 10)$ & 21.7 \\
\hline Education level & primary school only & 39.3 & Vitamin D supplement & use $(\geq 1)$ & 19.4 \\
\hline
\end{tabular}

$A T C$ anatomic therapeutic chemical classification, $B M I$ body mass index, $I Q R$ interquartile range, $M M S E$ mini mental state examination, $M N A$ mini nutrition assessment, 25(OH)D 25-hydroxyvitamin D

${ }^{\text {a } B e t w e e n ~} 1$ August 2011-31 December 2013

${ }^{\mathrm{b}}$ In a subpopulation aged $\geq 65$ years, between 21 September 2011-31 December 2013

${ }^{\mathrm{c}}$ Alcohol consumption index according to Garretsen [14]

${ }^{\mathrm{d}}$ All ATC-coded substances (ATC-coded supplements included)

After further adjustment for BMI, MMSE score, and use of multivitamins, statistical significance of associations with oral antidiabetics, metformin, and SSRIs disappeared. The inverse association with vitamin K antagonists was only borderline significant in subjects with a $\mathrm{BMI} \geq 27.0 \quad(\beta=-0.5, p=0.07)$. An inverse association with thiazide diuretics became statistically significant for subjects with a BMI $<21.0(\beta=-1.5, p=0.01)$, just as a positive association for angiotensin- 2 antagonists in patients with MMSE scores between 0-24 $(\beta=0.5$, $p=0.02)$. The most extreme differences in adjusted mean $25(\mathrm{OH}) \mathrm{D}$ level were $-19.3 \mathrm{nmol} / 1$ for thiazide diuretics and $+16.8 \mathrm{nmol} / \mathrm{l}$ for NSMRIs.

\section{Discussion}

Our study population consisted of 783 community-living older people who visited the geriatric outpatient clinic for various complaints. In the total population, polypharmacy and vitamin D deficiency were highly prevalent. Amongst users of vitamin D supplements, vitamin $\mathrm{D}$ deficiency was still prevalent. In patients not using supplemental vitamin $\mathrm{D}$, inverse associations with serum $25(\mathrm{OH})$ were observed for the number of medications used, (severe) polypharmacy, antidiabetics, cardiac glycosides, diuretics, ACE inhibitors, and SSRIs; positive associations for angiotensin-2 antagonists and NSMRIs.

In the following discussion of possible mechanisms behind observed associations, confounding by indication should be kept in mind: the entanglement of medication and indication for its prescription as precipitating factors.

To start with the inverse associations between the number of medications used and polypharmacy and vitamin D level, these were also observed in the only other cross-sectional study investigating these relationships [18]. Such inverse associations were not unexpected, as polypharmacy is associated with frailty [21], which in turn is a predictor of low circulating $25(\mathrm{OH}) \mathrm{D}$ [22].

A lack of relationship with use of PPIs was confirmed by a quasi-experimental study $(n=21)$ [23] and a prospective cohort study $(\mathrm{n}=58)$ [24]. A crosssectional study $(n=737)$ detected a borderline significant inverse association for which no explanation was given [18].

The absence of a significant association for osmotically acting laxatives could not be compared with literature data as no other publications were identified. 
Table 2 Medication ${ }^{\mathrm{a}}$ use and associated serum 25(OH)D, adjusted for age and gender ${ }^{\mathrm{b}}$, in 631 Dutch geriatric outpatients not using a specific vitamin D supplement

\begin{tabular}{|c|c|c|c|c|c|c|c|}
\hline \multicolumn{2}{|c|}{ Medication use } & \multirow[b]{2}{*}{$\mathrm{Use}^{\mathrm{c}}$} & \multirow[b]{2}{*}{ No. } & \multicolumn{4}{|c|}{ Serum $25(\mathrm{OH}) \mathrm{D}$} \\
\hline ATC code & Medication & & & $\begin{array}{l}\text { Mean }^{\mathrm{d}} \\
25(\mathrm{OH}) \mathrm{D} \\
(\mathrm{nmol} / \mathrm{l})\end{array}$ & {$[95 \% \mathrm{CI}]$} & $\begin{array}{l}\text { Difference }^{\mathrm{e}} \\
\text { in mean } \\
\text { V-25(OH)D }\end{array}$ & $p$ value \\
\hline Any & Number of medications used & & 631 & & & $-0.0^{\mathrm{f}}$ & 0.02 \\
\hline Any & Polypharmacy ${ }^{\mathrm{g}}$ & $\begin{array}{l}0 \\
1\end{array}$ & $\begin{array}{l}256 \\
375\end{array}$ & $\begin{array}{l}48.9 \\
45.2\end{array}$ & $\begin{array}{l}{[46.0 ; 52.0]} \\
{[42.9 ; 47.7]}\end{array}$ & -0.3 & 0.06 \\
\hline \multirow[t]{3}{*}{ Any } & Severe polypharmacy ${ }^{\mathrm{h}}$ & & & & & & \\
\hline & males & $\begin{array}{l}0 \\
1\end{array}$ & $\begin{array}{l}221 \\
47\end{array}$ & $\begin{array}{l}50.7 \\
41.0\end{array}$ & $\begin{array}{l}{[47.8 ; 53.7]} \\
{[35.5 ; 46.9]}\end{array}$ & -0.7 & 0.01 \\
\hline & females & $\begin{array}{l}0 \\
1\end{array}$ & $\begin{array}{l}306 \\
57\end{array}$ & $\begin{array}{l}45.3 \\
44.0\end{array}$ & $\begin{array}{l}{[42.5 ; 48.2]} \\
{[37.8 ; 50.8]}\end{array}$ & -0.1 & 0.72 \\
\hline $\mathrm{A} 02 \mathrm{BC}$ & Proton pump inhibitors & $\begin{array}{l}0 \\
1\end{array}$ & $\begin{array}{l}398 \\
233\end{array}$ & $\begin{array}{l}47.4 \\
45.5\end{array}$ & $\begin{array}{l}{[45.1 ; 49.9]} \\
{[42.5 ; 48.6]}\end{array}$ & -0.1 & 0.32 \\
\hline $\mathrm{A} 06 \mathrm{AD}$ & Osmotically acting laxatives & $\begin{array}{l}0 \\
1\end{array}$ & $\begin{array}{l}562 \\
69\end{array}$ & $\begin{array}{l}47.1 \\
43.7\end{array}$ & $\begin{array}{l}{[45.1 ; 49.1]} \\
{[38.4 ; 49.4]}\end{array}$ & -0.3 & 0.26 \\
\hline $\mathrm{A} 10 \mathrm{~B}$ & Oral antidiabetics & $\begin{array}{l}0 \\
1\end{array}$ & $\begin{array}{l}520 \\
111\end{array}$ & $\begin{array}{l}47.8 \\
41.9\end{array}$ & $\begin{array}{l}{[45.7 ; 49.9]} \\
{[37.8 ; 46.2]}\end{array}$ & -0.4 & 0.02 \\
\hline A10BA & Biguanides (metformin only) & $\begin{array}{l}0 \\
1\end{array}$ & $\begin{array}{l}541 \\
90\end{array}$ & $\begin{array}{l}47.7 \\
40.8\end{array}$ & $\begin{array}{l}{[45.7 ; 49.8]} \\
{[36.3 ; 45.5]}\end{array}$ & -0.5 & 0.01 \\
\hline \multirow[t]{4}{*}{ A10BB } & $\begin{array}{l}\text { Sulfonamides and urea derivatives } \\
\text { males, age }<80 \text { years }^{\mathrm{i}}\end{array}$ & $\begin{array}{l}0 \\
1\end{array}$ & $\begin{array}{l}148 \\
20\end{array}$ & $\begin{array}{l}54.9 \\
40.4\end{array}$ & $\begin{array}{l}{[51,3 ; 58,6]} \\
{[32.4 ; 49.3]}\end{array}$ & -1.1 & $<0.01$ \\
\hline & age $\geq 80$ years & $\begin{array}{l}0 \\
1\end{array}$ & $\begin{array}{l}90 \\
10\end{array}$ & $\begin{array}{l}42.6 \\
40.4\end{array}$ & $\begin{array}{l}{[38.3 ; 47.2]} \\
{[28.5 ; 54.6]}\end{array}$ & -0.2 & 0.76 \\
\hline & females, age $<80$ years & $\begin{array}{l}0 \\
1\end{array}$ & $\begin{array}{l}167 \\
19\end{array}$ & $\begin{array}{l}50.8 \\
48.2\end{array}$ & $\begin{array}{l}{[46.8 ; 55.0]} \\
{[37.2 ; 60.7]}\end{array}$ & -0.2 & 0.69 \\
\hline & age $\geq 80$ years & $\begin{array}{l}0 \\
1\end{array}$ & $\begin{array}{l}163 \\
14\end{array}$ & $\begin{array}{l}39.1 \\
48.0\end{array}$ & $\begin{array}{l}{[35.6 ; 42.7]} \\
{[35.5 ; 62.4]}\end{array}$ & 0.7 & 0.19 \\
\hline B01AA & Vitamin $\mathrm{K}$ antagonists & $\begin{array}{l}0 \\
1\end{array}$ & $\begin{array}{l}551 \\
80\end{array}$ & $\begin{array}{l}47.6 \\
41.1\end{array}$ & $\begin{array}{l}{[45.6 ; 49.6]} \\
{[36.3 ; 46.3]}\end{array}$ & -0.5 & 0.02 \\
\hline $\mathrm{B} 01 \mathrm{AC}$ & Platelet aggregation inhibitors & $\begin{array}{l}0 \\
1\end{array}$ & $\begin{array}{l}404 \\
227\end{array}$ & $\begin{array}{l}46.6 \\
46.9\end{array}$ & $\begin{array}{l}{[44.3 ; 49.0]} \\
{[43.8 ; 50.1]}\end{array}$ & 0.0 & 0.88 \\
\hline C01AA & Cardiac glycosides (digoxin only) & $\begin{array}{l}0 \\
1\end{array}$ & $\begin{array}{l}607 \\
24\end{array}$ & $\begin{array}{l}47.1 \\
38.2\end{array}$ & $\begin{array}{l}{[45.2 ; 49.0]} \\
{[29.9 ; 47.5]}\end{array}$ & -0.7 & 0.07 \\
\hline C03AA & Thiazide diuretics & $\begin{array}{l}0 \\
1\end{array}$ & $\begin{array}{l}516 \\
115\end{array}$ & $\begin{array}{l}46.8 \\
46.3\end{array}$ & $\begin{array}{l}{[44.8 ; 48.9]} \\
{[42.0 ; 50.8]}\end{array}$ & 0.0 & 0.82 \\
\hline \multirow[t]{2}{*}{$\mathrm{C} 03 \mathrm{CA}$} & $\begin{array}{l}\text { Loop diuretics } \\
\text { age }<80 \text { years }\end{array}$ & $\begin{array}{l}0 \\
1\end{array}$ & $\begin{array}{l}324 \\
30\end{array}$ & $\begin{array}{l}52.0 \\
48.5\end{array}$ & $\begin{array}{l}{[49.3 ; 54.8]} \\
{[40.2 ; 57.5]}\end{array}$ & -0.3 & 0.45 \\
\hline & age $\geq 80$ years & $\begin{array}{l}0 \\
1\end{array}$ & $\begin{array}{l}217 \\
60\end{array}$ & $\begin{array}{l}42.5 \\
34.4\end{array}$ & $\begin{array}{l}{[39.6 ; 45.7]} \\
{[29.4 ; 39.8]}\end{array}$ & -0.7 & 0.01 \\
\hline \multirow[t]{2}{*}{$\mathrm{C} 03 \mathrm{D}$} & $\begin{array}{l}\text { Potassium-sparing diuretics } \\
\text { males }\end{array}$ & $\begin{array}{l}0 \\
1\end{array}$ & $\begin{array}{l}256 \\
12\end{array}$ & $\begin{array}{l}49.6 \\
35.6\end{array}$ & $\begin{array}{l}{[46.9 ; 52.3]} \\
{[25.8 ; 47.1]}\end{array}$ & -1.1 & 0.02 \\
\hline & females & $\begin{array}{l}0 \\
1\end{array}$ & $\begin{array}{l}332 \\
31\end{array}$ & $\begin{array}{l}45.1 \\
45.0\end{array}$ & $\begin{array}{l}{[42.5 ; 47.9]} \\
{[36.5 ; 54.3]}\end{array}$ & 0.0 & 0.98 \\
\hline $\mathrm{C} 07 \mathrm{AB}$ & Selective beta-blocking agents & $\begin{array}{l}0 \\
1\end{array}$ & $\begin{array}{l}441 \\
190\end{array}$ & $\begin{array}{l}47.1 \\
45.9\end{array}$ & $\begin{array}{l}{[44.8 ; 49.4]} \\
{[42.6 ; 49.4]}\end{array}$ & -0.1 & 0.60 \\
\hline $\mathrm{C} 08 \mathrm{CA}$ & Dihydropyridines & $\begin{array}{l}0 \\
1\end{array}$ & $\begin{array}{l}534 \\
97\end{array}$ & $\begin{array}{l}46.7 \\
46.9\end{array}$ & $\begin{array}{l}{[44.7 ; 48.7]} \\
{[42.3 ; 51.8]}\end{array}$ & 0.0 & 0.92 \\
\hline C09AA & ACE inhibitors & $\begin{array}{l}0 \\
1\end{array}$ & $\begin{array}{l}474 \\
157\end{array}$ & $\begin{array}{l}47.9 \\
43.3\end{array}$ & $\begin{array}{l}{[45.7 ; 50.1]} \\
{[39.7 ; 47.0]}\end{array}$ & -0.3 & 0.04 \\
\hline
\end{tabular}


Table 2 (continued)

\begin{tabular}{|c|c|c|c|c|c|c|c|}
\hline \multicolumn{2}{|c|}{ Medication use } & \multirow[b]{2}{*}{$\mathrm{Use}^{\mathrm{c}}$} & \multirow[b]{2}{*}{ No. } & \multicolumn{4}{|c|}{ Serum $25(\mathrm{OH}) \mathrm{D}$} \\
\hline ATC code & Medication & & & $\begin{array}{l}\text { Mean }^{\mathrm{d}} \\
\text { 25(OH)D } \\
(\mathrm{nmol} / \mathrm{l})\end{array}$ & {$[95 \% \mathrm{CI}]$} & $\begin{array}{l}\text { Difference }^{\mathrm{e}} \\
\text { in mean } \\
\mathrm{V}-25(\mathrm{OH}) \mathrm{D}\end{array}$ & $p$ value \\
\hline C09CA & Angiotensin-2 antagonists & $\begin{array}{l}0 \\
1\end{array}$ & $\begin{array}{l}524 \\
107\end{array}$ & $\begin{array}{l}46.1 \\
49.6\end{array}$ & $\begin{array}{l}{[44.1 ; 48.2]} \\
{[45.0 ; 54.4]}\end{array}$ & 0.3 & 0.18 \\
\hline C10AA & Statins & $\begin{array}{l}0 \\
1\end{array}$ & $\begin{array}{l}451 \\
180\end{array}$ & $\begin{array}{l}47.4 \\
45.1\end{array}$ & $\begin{array}{l}{[45.2 ; 49.6]} \\
{[41.7 ; 48.6]}\end{array}$ & -0.2 & 0.28 \\
\hline N02BE & Anilides (paracetamol only) & $\begin{array}{l}0 \\
1\end{array}$ & $\begin{array}{l}538 \\
93\end{array}$ & $\begin{array}{l}47.3 \\
43.3\end{array}$ & $\begin{array}{l}{[45.3 ; 49.4]} \\
{[38.7 ; 48.1]}\end{array}$ & -0.3 & 0.13 \\
\hline $\mathrm{N} 05^{\mathrm{j}}$ & Benzodiazepines & $\begin{array}{l}0 \\
1\end{array}$ & $\begin{array}{l}485 \\
146\end{array}$ & $\begin{array}{l}46.5 \\
47.3\end{array}$ & $\begin{array}{l}{[44.4 ; 48.7]} \\
{[43.5 ; 51.4]}\end{array}$ & 0.1 & 0.73 \\
\hline N06A & $\begin{array}{l}\text { Antidepressants } \\
\text { males }\end{array}$ & $\begin{array}{l}0 \\
1\end{array}$ & $\begin{array}{l}233 \\
35\end{array}$ & $\begin{array}{l}48.6 \\
51.4\end{array}$ & $\begin{array}{l}{[45.8 ; 51.4]} \\
{[44.0 ; 59.3]}\end{array}$ & 0.2 & 0.49 \\
\hline & females & $\begin{array}{l}0 \\
1\end{array}$ & $\begin{array}{l}296 \\
67\end{array}$ & $\begin{array}{l}45.8 \\
42.1\end{array}$ & $\begin{array}{l}{[43.0 ; 48.8]} \\
{[36.4 ; 48.1]}\end{array}$ & -0.3 & 0.26 \\
\hline N06AA & $\begin{array}{l}\text { NSMRIs } \\
\text { age }<80 \text { years }\end{array}$ & $\begin{array}{l}0 \\
1\end{array}$ & $\begin{array}{l}333 \\
21\end{array}$ & $\begin{array}{l}50.8 \\
68.3\end{array}$ & $\begin{array}{l}{[48,2 ; 53,4]} \\
{[56.7 ; 80.9]}\end{array}$ & 1.1 & $<0.00$ \\
\hline & age $\geq 80$ years & $\begin{array}{l}0 \\
1\end{array}$ & $\begin{array}{l}254 \\
23\end{array}$ & $\begin{array}{l}40.8 \\
39.1\end{array}$ & $\begin{array}{l}{[38.1 ; 43.7]} \\
{[30.5 ; 48,8]}\end{array}$ & -0.1 & 0.72 \\
\hline N06AB & SSRIs & $\begin{array}{l}0 \\
1\end{array}$ & $\begin{array}{l}578 \\
53\end{array}$ & $\begin{array}{l}47.4 \\
39.8\end{array}$ & $\begin{array}{l}{[45.4 ; 49.4]} \\
{[34.1 ; 46.0]}\end{array}$ & -0.6 & 0.02 \\
\hline
\end{tabular}

ATC anatomic therapeutic chemical Classification, CI confidence interval, PPIs proton pump inhibitors, NSMRIs non-selective monoamine reuptake inhibitors, SSRIs selective serotonin reuptake inhibitors, 25(OH)D 25-hydroxyvitamin D

${ }^{\mathrm{a}}$ ATC-coded substances

${ }^{\mathrm{b}}$ If interaction with medication: stratification

${ }^{\mathrm{c}} 0=$ no use, $1=$ use

${ }^{\mathrm{d}}$ Squared mean of 'square-root transformed 25(OH)D'

${ }^{e}$ Difference in mean 'square root transformed 25(OH)D' between users and non-users of a medication (which is equivalent to the regression coefficient $\beta$ of an association between drug use and 'square root transformed 25(OH)D')

${ }^{\mathrm{f}}-0.043=$ regression coefficient $\beta$ of the association between the number of medications used and 'square root transformed 25(OH)D' (which is equivalent to the difference in mean 'square root transformed $25(\mathrm{OH}) \mathrm{D}$ ' between users and non-users of a medication)

${ }^{\mathrm{g}}$ Use of $\geq 5$ medications concomitantly

${ }^{\mathrm{h}}$ Use of $\geq 10$ medications concomitantly

${ }^{\mathrm{i}}$ Independent $t$ test because of unequal variances

${ }^{\mathrm{j}} \mathrm{N} 05 \mathrm{BA}, \mathrm{N} 05 \mathrm{CD}$ or N05CF

In three cross-sectional studies a negative association with oral antidiabetics was also observed $(n=737$, $n=407, N=11256)[18,25,26]$. At the same time, a previous quasi-experimental study $(n=19)$ and a crosssectional study $(n=698)$, did not show these associations [27, 28]. Obviously, not only medication itself might be an explanation, but also the indication for its prescription. This confounding by disease is supported by a meta-analysis of prospective studies investigating 25(OH)D and type 2 diabetes [29]. Underlying mechanisms might include a decreased insulin sensitivity related with increased parathyroid hormone levels which, in turn, are associated with vitamin D deficiency [30].
Another explanation might be vitamin D deficiency being mediated by inflammatory mediators which are associated with the presence of excess visceral fat, a risk factor for diabetes mellitus type-2 [10].

Lower 25(OH)D levels in users of vitamin $K$ antagonists were also seen in a Dutch cross-sectional study in 514 females;[18] and in a German cross-sectional study in 7553 males [26]. In three other cross-sectional studies $(n=116$, $n=48, n=127)$ and one prospective cohort study $(n=167)$, no associations were observed [31-34].

Associations with platelet aggregation inhibitors were contradictory in literature. Similar to our study, one cross-sectional study $(n=1301, n=737)$ did not show an 
inverse relationship, [18] while three others did $(n=11256$, $n=2016, n=459)[26,35,36]$. Confounding by indication might lie behind the suggestion that elevated inflammatory markers link hypovitaminosis D with increased risk of cardiovascular disease [36].

Opposite to our results, an inverse relationship with cardiac glycosides was not observed in two cross-sectional studies investigating digoxin $(n=1301, n=11256)[18,26]$.

Also in other studies observed associations with thiazide diuretics were mixed. Two cross-sectional studies $(n=1301$, $n=737 ; n=66$ ) did not report any significant association [18, 37]. Contrarily, a small randomised placebo-controlled crossover trial $(n=23)$ reported a dose-dependent increase in $25(\mathrm{OH}) \mathrm{D}$ levels, [38] while a third cross-sectional study $(n=302)$ reported an inverse association [39]. This might be confounded by indication as hypertension is inversely associated with vitamin D status [40].

Lower 25(OH)D levels amongst users of loop diuretics were also determined in two cross-sectional studies $(n=1301, n=280),[18,41]$ In the first study, results were significant when adjusted for age and gender; when number of chronic diseases was added, significance disappeared. However, in a complex model with six extra covariates the association was again significant. In two other cross-sectional studies $(n=302, n=77)$ associations were not statistically significant [39, 42].

Our observation of an inverse association with potassiumsparing diuretics was not supported by one other crosssectional study $(n=1301)$ [18]

No association with selective beta-blocking agents was found in two cohorts of a cross-sectional study $(n=1301$, $n=737$ ) [18].

Similar to our results, no difference in $25(\mathrm{OH}) \mathrm{D}$ levels was reported in a small cross-sectional study $(n=22)$ investigating dihydropyridines [43]. This was also the case in two other, larger cross-sectional studies $(n=1301, n=11256)$ investigating the broader therapeutic subgroup of calcium blocking agents $[18,26]$. However, in a second cohort of one of these studies $(n=737)$ a statistically significant inverse association was identified [18]. It is hypothesised that activation of the nuclear pregnane $\mathrm{X}$ receptor by calcium blockers might lead to catabolism of $25(\mathrm{OH}) \mathrm{D}$ [44].

Lower 25(OH)D levels in users of $A C E$ inhibitors were also observed in a German and a Dutch cross-sectional study ( $n=11256, n=737)$ [ref 15, 22], while in a second cohort of the Dutch study $(n=1301)$ [18] and in two quasi-experimental studies $(n=73, n=60)[45,46]$ no relationship was shown. A third quasi-experimental study reported higher levels in users of quinapril $(n=23)$ and no change in users of enalapril $(n=23)$ [47]. It cannot be excluded that an inverse association reflects the inverse relationship between low $25(\mathrm{OH}) \mathrm{D}$ level and the indication for prescription: high blood pressure ${ }^{29}$ and associated comorbidities of diabetes [29].
Our finding of a positive association with angiotensin-2 antagonists was not detected in two other cross-sectional studies $(n=11256, n=31)[26,48]$.

Similar to our study, no association with statins was detected in two regression models of a cross-sectional study after multiple adjustments $(n=737)$; in a third model of this study, a borderline significantly inverse association was found after adjustment for age and gender only [18]. In a large cross-sectional and small cohort study $(n=11256, n=208)$ associations observed were positive $[26,49]$. Studies investigating individual statins reported null or positive associations [9]. Several mechanisms are suggested for a positive association: an increase in level of precursors for vitamin $\mathrm{D}$ synthesis in the skin by inhibition of the HMG-CoA reductase, [50] competition of statins with the metabolising enzyme CYP3A4, [51], or inhibition by statins of vitamin D-consuming inflammatory processes [27].

The absence of a significant association with benzodiazepines was also reported in two other cross-sectional studies $(n=737$, $n=589)[18,52]$. In a second cohort of the first study, a significant inverse association was observed $(n=1301)$ [18].

The finding of an inverse relationship with antidepressants in one other cross-sectional study $(n=589)$ [52] supports our finding of lower 25(OH)D levels in users of SSRIs. An inverse association might be explained by inhibition of 25(OH)D synthesis through inhibition of the CYP3A4 enzyme [53]. The inverse relationship between depression and 25(OH)D reported in literature [54] does not match with our results for users of NSMRIs, who had higher 25(OH)D levels compared to nonusers in the complex model.

The finding that a considerable part of the reported users of vitamin D supplements were still deficient is noteworthy. Possible explanations could lie in the type and quality of vitamin D supplements, dosing guidelines, duration of use, compliance, and interindividual differences in pharmacodynamics and pharmacokinetics in our group of patients, but these remain to be investigated further.

Major limitation of our study is the cross-sectional design, which implies that no conclusions can be drawn about causal relationships. A critical remark has also to be made about our interpretation of cross-sectional. Laboratory measurements of $25(\mathrm{OH}) \mathrm{D}$ were included until four months preceding the first visit of the outpatient clinic; $95 \%$ of these assays were measured on the day of first visit. Underlying considerations were the following. Firstly, the pragmatic reason that physicians did not request vitamin $\mathrm{D}$ testing within four months following a previous measurement in the hospital. Secondly, the assumption that the investigated drugs were already being used for a longer period, so also at the moment of a previous vitamin D assessment. One more critical remark must be made, on the HPLC assay used for $25(\mathrm{OH}) \mathrm{D}$ measurement. This is a less accurate method compared to the golden standard liquid chromatography-mass spectrometry. Another limitation of the study is the registration of the drugs used, which was 
dependent on the cooperation, memory, and assistance of the patients or their accompanying persons, and the accurateness of drug registration by pharmacies. Furthermore, although patients were also routinely asked for drug therapy compliance, no drug analysis was undertaken to confirm their response or that of their helper. This, combined with the fact that registration in the patient files was not standardised, was reason not to include compliance as a potential covariate. Uncertainty about drug compliance also influences the effect of use of a vitamin D supplement. Long-term formulations could be an option to improve this. Also important to mention is that we did not adjust for confounding by indication. Reasons were the complexity of this confounder and lacking data. Finally, we note that one or more of the statistically significant results may be regarded as chance findings. The large number of medication groups increases the possibility of false-positive results.

Despite these reservations, our study has several noteworthy strengths. It adds to the data of the relatively unexplored field of drug-nutrient interactions and reflects the typical population of community-dwelling older people visiting a nonacademic regional hospital. That we investigated a large number of drug groups which are frequently used by older people further contributes to the quality of the study. Finally, the ATC-coding is a major strength; it enabled us to investigate drug and supplement use in a systematic way. Moreover, as far as we know, in studies investigating drug use and circulating $25(\mathrm{OH}) \mathrm{D}$, we are the first to discriminate between users and non-users of supplemental vitamin D.

\section{Conclusion}

Drug use should be recognised as a determinant of vitamin D status. The still high prevalence of vitamin D deficiency in subjects using a vitamin D supplement requires further investigation.

Acknowledgments We would like to acknowledge Prof. Dr. Hendriek Boshuizen and Dr. Janette de Goede of Wageningen University for their expert advice on statistics, Dr. Elske Brouwer-Brolsma of Wageningen University for her advice on epidemiological issues, and Dr. Jacqueline Klein-Gunnewiek, Dr. Silvia Endenburg, and Dr. Michiel Balvers of the laboratory of the Gelderse Vallei Hospital in Ede for their support in laboratory issues. Also, we would like to thank the medical staff, nurses, and secretarial employees of the hospital geriatric department, information specialists of the hospital library, and students of the division of human nutrition of Wageningen University for their contributions in other theoretical and practical matters related to this study.

Compliance with ethical standards According to the Dutch Medical Research Involving Human Subjects Act (WMO) no ethical approval was needed, since this study involved retrospective analysis of anonymised patient data only.

Conflict of interest The authors declare that they have no conflicts of interest.
Authors' contributions AvO: acquisition, analysis and interpretation of data, preparation, revision, and editing of manuscript. AJ: study concept and design, acquisition and interpretation of data, review and approval of manuscript. RD: analysis and interpretation of data, review and approval of manuscript. RW: study concept and design, interpretation of data, review and approval of manuscript.

Open Access This article is distributed under the terms of the Creative Commons Attribution 4.0 International License (http:// creativecommons.org/licenses/by/4.0/), which permits unrestricted use, distribution, and reproduction in any medium, provided you give appropriate credit to the original author(s) and the source, provide a link to the Creative Commons license, and indicate if changes were made. References

1. Bouvy J, De Bruin M, Koopmanschap M (2015) Epidemiology of adverse drug reactions in Europe: a review of recent observational studies. Drug Saf 38(5):437-453

2. Onder G, Petrovic M, Tangiisuran B, Meinardi MC, MarkitoNotenboom WP, Somers A, Rajkumar C, Bernabei R, van der Cammen TJ (2010) Development and validation of a score to assess risk of adverse drug reactions among in-hospital patients 65 years or older: the GerontoNet ADR risk score. Arch Intern Med 170(13): $1142-1148$

3. van Schoor NM, Lips P (2011) Worldwide vitamin D status. Best Pract Res Clin Endocrinol Metab 25(4):671-680

4. Autier P, Boniol M, Pizot C, Mullie P (2014) Vitamin D status and ill health: a systematic review. Lancet Diabetes Endocrinol 2(1):76-89

5. Cannell JJ, Grant WB, Holick MF (2014) Vitamin D and inflammation. Dermato-Endocrinology 6(1):e983401

6. MacLaughlin J, Holick MF (1985) Aging decreases the capacity of human skin to produce vitamin D3. J Clin Invest 76(4):1536-1538

7. Cashman KD, Fitzgerald AP, Kiely M, Seamans KM (2011) A systematic review and meta-regression analysis of the vitamin D intake-serum 25-hydroxyvitamin D relationship to inform European recommendations. Br J Nutr 106(11):1638-1648

8. Prentice A, Goldberg GR, Schoenmakers I (2008) Vitamin D across the lifecycle: physiology and biomarkers. Am J Clin Nutr 88(2): 500S-506S

9. Fornari R, Francomano D, Greco EA, Marocco C, Lubrano C, Wannenes F, Papa V, Bimonte VM, Donini LM, Lenzi A, Aversa A, Migliaccio S (2015) Lean mass in obese adult subjects correlates with higher levels of vitamin $\mathrm{D}$, insulin sensitivity and lower inflammation. J Endocr Invest 38(3):367-372

10. Ding C, Parameswaran V, Blizzard L, Burgess J, Jones G (2010) Not a simple fat-soluble vitamin: Changes in serum 25-(OH)D levels are predicted by adiposity and adipocytokines in older adults. J Intern Med 268(5):501-510

11. van Orten-Luiten AC, Janse A, Dhonukshe-Rutten RA, Witkamp RF (2014) The association between drugs frequently used by the elderly and vitamin D blood levels: a review of observational and experimental studies. Drugs Aging 31(2):111-123

12. Boullata JI, Hudson LM (2012) Drug-nutrient interactions: a broad view with implications for practice. J Acad Nutr Diet 112(4):506-517

13. 13Coelho T, Paúl C, Gobbens RJJ, Fernandes L (2015) Determinants of frailty: the added value of assessing medication. Front Aging Neurosci 7, art 56: 1-7

14. 14Boulos C, Salameh P, Barberger-Gateau P (2015) Malnutrition and frailty in community dwelling older adults living in a rural setting. Clin Nutr: (in press) 
15. Semba RD, Bartali B, Zhou J, Blaum C, Ko CW, Fried LP (2006) Low serum micronutrient concentrations predict frailty among older women living in the community. J Gerontol A Biol Sci Med Sci 61(6):594-599

16. Fried LP, Ferrucci L, Darer J, Williamson JD, Anderson G (2004) Untangling the concepts of disability, frailty, and comorbidity: implications for improved targeting and care. J Gerontol A Biol Sci Med Sci 59(3):255-263

17. Garretsen HFL, Knibbe RA (1983) Alkohol prevalentie onderzoek Rotterdam/Limburg: Landelijk Eindrapport. Ministerie van Welzijn Volksgezondheid en Cultuur, Leidschendam (in Dutch)

18. Sohl E, van Schoor NM, de Jongh RT, de Vries OJ, Lips P (2012) The impact of medication on vitamin D status in older individuals. Eur J Endocrinol 166(3):477-485

19. Brouwer-Brolsma EM, van de Rest O, Tieland M, van der Zwaluw NL, Steegenga WT, Adam JJ, van Loon LJ, Feskens EJ, de Groot LC (2013) Serum 25-hydroxyvitamin D is associated with cognitive executive function in Dutch prefrail and frail elderly: a crosssectional study exploring the associations of 25-hydroxyvitamin D with glucose metabolism, cognitive performance and depression. J Am Med Dir Assoc 14(11):852.e9-852.e17

20. Bland JM, Altman DG (1996) Statistics notes: the use of transformation when comparing two means. BMJ 312(7039):1153

21. Herr M, Robine J-M, Pinot J, Arvieu J-J, Ankri J (2015) Polypharmacy and frailty: prevalence, relationship, and impact on mortality in a French sample of 2350 old people. Pharmacoepidem Drug Saf 24(6):637-646

22. Vogt S, Decke S, de las Heras Gala T, Linkohr B, Koenig W, Ladwig K-H, Peters A, Thorand B (2015) Prospective association of vitamin D with frailty status and all-cause mortality in older adults: results from the KORA-Age Study. Prev Med 73:40-46

23. Hansen KE, Jones AN, Lindstrom MJ, Davis LA, Ziegler TE, Penniston KL, Alvig AL, Shafer MM (2010) Do proton pump inhibitors decrease calcium absorption? J Bone Miner Res 25(12): 2786-2795

24. Sharara AI, El-Halabi MM, Ghaith OA, Habib RH, Mansour NM, Malli A, El Hajj-Fuleihan G (2012) Proton pump inhibitors have no measurable effect on calcium and bone metabolism in healthy young males: a prospective matched controlled study. Metabolism 62(4):518-526

25. Suzuki A, Kotake M, Ono Y, Kato T, Oda N, Hayakawa N, Hashimoto S, Itoh M (2006) Hypovitaminosis D in type 2 diabetes mellitus: association with microvascular complications and type of treatment. Endocr J 53(4):503-510

26. Ernst JB, Kuhn J, Becker T, Dreier J, Börgermann J, Knabbe C, Gummert JF, Zittermann A (2015) Association between circulating 25-hydroxyvitamin $\mathrm{D}$ levels and medication use in patients scheduled for cardiac surgery. Nutri Metab Cardiovasc Dis 25(3):280-286

27. Sathyapalan T, Shepherd J, Arnett C, Coady AM, Kilpatrick ES, Atkin SL (2010) Atorvastatin increases 25-hydroxy vitamin D concentrations in patients with polycystic ovary syndrome. Clin Chem 56(11):1696-1700

28. Kos E, Liszek MJ, Emanuele MA, Durazo-Arvizu R, Camacho P (2012) Effect of metformin therapy on vitamin D and vitamin B12 levels in patients with type 2 diabetes mellitus. Endocr Pract 18(2): 179-184

29. Forouhi NG, Ye Z, Rickard AP, Khaw KT, Luben R, Langenberg C, Wareham NJ (2012) Circulating 25-hydroxyvitamin D concentration and the risk of type 2 diabetes: results from the European Prospective Investigation into Cancer (EPIC)-Norfolk cohort and updated meta-analysis of prospective studies. Diabetologia 55(8): 2173-2182

30. Tai K, Need AG, Horowitz M, Chapman IM (2008) Vitamin D, glucose, insulin, and insulin sensitivity. Nutrition 24(3):279-285
31. Stenova E, Steno B, Killinger Z, Baqi L, Payer J (2011) Effect of long-term oral anticoagulant therapy on bone mineral density and bone turnover markers: a prospective 12 month study. Bratisl Lek Listy 112(2):71-76

32. Avgeri M, Papadopoulou A, Platokouki H, Douros K, Rammos S, Nicolaidou P, Aronis S (2008) Assessment of bone mineral density and markers of bone turnover in children under long-term oral anticoagulant therapy. J Pediatr Hematol Oncol 30(8):592-597

33. Sato Y, Honda Y, Kunoh H, Oizumi K (1997) Long-term oral anticoagulation reduces bone mass in patients with previous hemispheric infarction and nonrheumatic atrial fibrillation. Stroke 28(12):2390-2394

34. Sato Y, Honda Y, Jun I (2010) Long-term oral anticoagulation therapy and the risk of hip fracture in patients with previous hemispheric infarction and nonrheumatic atrial fibrillation. Cerebrovasc Dis 29(1):73-78

35. Vestergaard P, Hermann P, Jensen JE, Eiken P, Mosekilde L (2012) Effects of paracetamol, non-steroidal anti-inflammatory drugs, acetylsalicylic acid, and opioids on bone mineral density and risk of fracture: results of the Danish Osteoporosis Prevention Study (DOPS). Osteoporos Int 23(4):1255-1265

36. Cigolini M, Iagulli MP, Miconi V, Galiotto M, Lombardi S, Targher G (2006) Serum 25-hydroxyvitamin D3 concentrations and prevalence of cardiovascular disease among type 2 diabetic patients. Diabetes Care 29(3):722-724

37. Perry HM 3rd, Jensen J, Kaiser FE, Horowitz M, Perry HM Jr, Morley JE (1993) The effects of thiazide diuretics on calcium metabolism in the aged. J Am Geriatr Soc 41(8):818-822

38. Rejnmark L, Vestergaard P, Pedersen AR, Heickendorff L, Andreasen F, Mosekilde L (2003) Dose-effect relations of loop- and thiazide-diuretics on calcium homeostasis: a randomized, double-blinded Latin-square multiple cross-over study in postmenopausal osteopenic women. Eur J Clin Invest 33(1):41-50

39. Drinka PJ, Krause PF, Nest LJ, Goodman BM (2007) Determinants of parathyroid hormone levels in nursing home residents. J Am Med Dir Assoc 8(5):328-331

40. Judd SE, Nanes MS, Ziegler TR, Wilson PW, Tangpricha V (2008) Optimal vitamin D status attenuates the ageassociated increase in systolic blood pressure in white Americans: results from the third National Health and Nutrition Examination Survey. Am J Clin Nutr 87(1):136141

41. Rejnmark L, Vestergaard P, Heickendorff L, Andreasen F, Mosekilde L (2005) Effects of long-term treatment with loop diuretics on bone mineral density, calcitropic hormones and bone turnover. J Intern Med 257(2):176-184

42. Reichel H, Deibert B, Geberth S, Schmidt-Gayk H, Ritz E (1992) Frusemide therapy and intact parathyroid hormone plasma concentrations in chronic renal insufficiency. Nephrol Dial Transplant $7(1): 8-15$

43. Albers MM, Johnson W, Vivian V, Jackson RD (1991) Chronic use of the calcium channel blocker nifedipine has no significant effect on bone metabolism in men. Bone 12(1):39-42

44. Grober U, Kisters K (2012) Influence of drugs on vitamin D and calcium metabolism. Dermatoendocrinol 4(2):158-166

45. Perez-Castrillon JL, Vega G, Abad L, Sanz A, Chaves J, Hernandez G, Duenas A (2007) Effects of atorvastatin on vitamin $\mathrm{D}$ levels in patients with acute ischemic heart disease. Am J Cardiol 99(7):903-905

46. Perez-Castrillon JL, Justo I, Sanz A, De Luis D, Duenas A (2006) Effect of angiotensin converting enzyme inhibitors on $1.25-(\mathrm{OH}) 2$ 
D levels of hypertensive patients. Relationship with ACE polymorphisms. Horm Metab Res 38(12):812-816

47. Perez-Castrillon JL, Silva J, Justo I, Sanz A, Martin-Luquero M, Igea R, Escudero P, Pueyo C, Diaz C, Hernandez G, Duenas A (2003) Effect of quinapril, quinapril-hydrochlorothiazide, and enalapril on the bone mass of hypertensive subjects: relationship with angiotensin converting enzyme polymorphisms. Am J Hypertens 16(6):453-459

48. Perez-Castrillon JL, De Luis D, Inglada L, Olmos Martinez JM, Pinacho F, Conde R, Gonzalez-Sagrado M, DuenasLaita A (2012) Telmisartan effects on remodelling bone markers in hypertensive patients. Nutr Hosp 27(1):276-280

49. Aloia JF, Li-Ng M, Pollack S (2007) Statins and vitamin D. Am J Cardiol 100(8):1329

50. Ott C, Raff U, Schneider MP, Titze SI, Schmieder RE (2013) 25Hydroxyvitamin D insufficiency is associated with impaired renal endothelial function and both are improved with rosuvastatin treatment. Clin Res Cardiol 102(4):299-304
51. Robien K, Oppeneer SJ, Kelly JA, Hamilton-Reeves JM (2013) Drug-vitamin D interactions: a systematic review of the literature. Nutr Clin Pract 28:194-208

52. Verhoeven V, Vanpuyenbroeck K, Lopez-Hartmann M, Wens J, Remmen R (2012) Walk on the sunny side of life-epidemiology of hypovitaminosis $\mathrm{D}$ and mental health in elderly nursing home residents. J Nutr Health Aging 16(4):417-420

53. Zhou SF, Xue CC, Yu XQ, Li C, Wang G (2007) Clinically important drug interactions potentially involving mechanismbased inhibition of cytochrome P450 3A4 and the role of therapeutic drug monitoring. Ther Drug Monit 29(6):687710

54. Milaneschi Y, Hoogendijk W, Lips P, Heijboer AC, Schoevers R, van Hemert AM, Beekman AT, Smit JH, Penninx BW (2013) The association between low vitamin D and depressive disorders. Mol Psychiatry 95(7):3225-3233 